\title{
A phase I study of the oral gamma secretase inhibitor R04929097 in combination with gemcitabine in patients with advanced solid tumors (PHL-078/CTEP 8575)
}

\author{
Suzanne Richter • Philippe L. Bedard • Eric Xueyu Chen • Blaise A. Clarke • Ben Tran • \\ Sebastien J. Hotte • Anastasios Stathis • Hal W. Hirte - Albiruni R. A. Razak • \\ Michael Reedijk • Zhuo Chen • Brenda Cohen • Wen-Jiang Zhang • Lisa Wang • \\ S. Percy Ivy • Malcolm J. Moore • Amit M. Oza • Lillian L. Siu • Elaine McWhirter
}

Received: 10 March 2013 / Accepted: 22 April 2013 /Published online: 5 May 2013

(C) The Author(s) 2013. This article is published with open access at Springerlink.com

\begin{abstract}
Summary Purpose To establish the recommended phase II dose of the oral $\gamma$-secretase inhibitor RO4929097 (RO) in combination with gemcitabine; secondary objectives include the evaluation of safety, tolerability, pharmacokinetics, biomarkers of Notch signaling and preliminary anti-tumor activity. Methods Patients with advanced solid tumors were enrolled in cohorts of escalating RO dose levels (DLs). Tested RO DLs were $20 \mathrm{mg}, 30 \mathrm{mg}, 45 \mathrm{mg}$ and $90 \mathrm{mg}$. RO was administered orally, once daily on days $1-3,8-10,15-17$, 22-24. Gemcitabine was administered at $1,000 \mathrm{mg} / \mathrm{m}^{2}$ on d1, 8 , and 15 in 28 d cycles. Dose limiting toxicities (DLTs) were assessed by CTCAE v4. Serial plasma was collected for RO (total and unbound) and gemcitabine pharmacokinetic
\end{abstract}

This study (PHL-078/CTEP 8575) is conducted by the Princess Margaret Hospital Phase I Consortium with support from the US National Cancer Institute U01 Cooperative Agreement Award (U01-CA132123)

S. Richter · P. L. Bedard • E. X. Chen · B. Tran • A. Stathis •

A. R. A. Razak - W.-J. Zhang • M. J. Moore · A. M. Oza $\cdot$ L. L. Siu

Princess Margaret Cancer Centre,

Drug Development Program, 610 University Avenue,

Toronto, Canada M5G 2M9

B. A. Clarke

Department of Pathology, University Health Network,

200 Elizabeth Street,

Toronto, Canada M5G 2C4

S. J. Hotte $\cdot$ H. W. Hirte $\cdot$ E. McWhirter $(\bowtie)$

Juravinski Cancer Centre, 699 Concession Street,

Hamilton, Canada L8V 5C2

e-mail: elaine.mcwhirter@jcc.hhsc.ca

M. Reedijk

Princess Margaret Cancer Centre, Department of Surgical

Oncology, University Health Network, 610 University Avenue,

Toronto, Canada M5G 2M9 analysis. Biomarkers of Notch signaling were assessed by immunohistochemistry in archival tissue. Antitumor activity was evaluated (RECIST 1.1). Results A total of 18 patients were enrolled to establish the recommended phase II dose. Of these, 3 patients received $20 \mathrm{mg}$ RO, 7 patients received $30 \mathrm{mg}$ RO, 6 patients received $45 \mathrm{mg}$ RO and 2 patients received $90 \mathrm{mg}$ RO. DLTs were grade 3 transaminitis (30 mg RO), grade 3 transaminitis and maculopapular rash (45 mg RO), and grade 3 transaminitis and failure to receive $75 \%$ of planned RO doses secondary to prolonged neutropenia (90 mg); all were reversible. The maximum tolerated dose was exceeded at $90 \mathrm{mg}$ RO. Pharmacokinetic analysis of both total and free RO confirmed the presence of autoinduction at

\section{Z. Chen}

Applied Molecular Profiling Laboratory, University Health

Network, 610 University Ave,

Toronto, ON, Canada M5G 2M9

B. Cohen

University Health Network, 200 Elizabeth Street,

Toronto, Canada M5G 2C4

L. Wang

Princess Margaret Cancer Centre, Department of Biostatistics, 610 University Avenue,

Toronto, ON, Canada M5G 2M9

S. P. Ivy

Cancer Evaluation Program, National Cancer Institute, Rockville, MD 20852-7426, USA 
45 and $90 \mathrm{mg}$. Median levels of Notch3 staining were higher in individuals who received fewer than 4 cycles $(p=0.029)$. Circulating angiogenic factor levels did not correlate with time to progression or $\geq$ grade 3 adverse events. Best response (RECIST 1.1) was partial response (nasopharyngeal cancer) and stable disease $>4$ months was observed in 3 patients (pancreas, tracheal, and breast primary cancers). Conclusions RO and gemcitabine can be safely combined. The recommended phase II dose

of RO was $30 \mathrm{mg}$ in combination with gemcitabine $1,000 \mathrm{mg} / \mathrm{m}^{2}$. Although RO exposure was limited by the presence of autoinduction, RO levels achieved exceeded the area under the concentration-time curve for $0-24 \mathrm{~h}\left(\mathrm{AUC}_{0-24}\right)$ predicted for efficacy in preclinical models using daily dosing. Evidence of clinical antitumor activity and prolonged stable disease were identified.

Keywords Phase I $\cdot$ RO4929097 · Notch inhibition · Gemcitabine

\section{Introduction}

Aberrations in the Notch signaling axis have been implicated in the uncontrolled growth of malignant cells [1,2]. Notch is a cell surface receptor comprised of four paralogues (Notch 14) and five canonical ligands including the Delta-like ligands (DLL-1, DLL-3, DLL-4 and Jagged 1 and 2). Ligand binding results in gamma-secretase induced intramembrane cleavage and subsequent formation of the Notch intracellular domain (NICD). NICD translocates into the nucleus and activates transcription of target genes including MYC and HES1. [3, 4]. The pathophysiologic effects of Notch activation include maintenance of a pluripotent stem cell-like state [5] and the promotion of angiogenesis [6, 7]. Inhibition of Notch signaling results in decreased tumor growth in solid tumor xenograft models, including pancreatic cancers [8].

Gamma secretase inhibition represents a novel approach to Notch signaling disruption. RO4929097 is a potent inhibitor of gamma secretase [9]. Intermittent and daily dosing in xenograft models demonstrates antitumor activity [9]. Evaluation of RO4929097 in a phase I study demonstrated good tolerability [10]. Common grade 1 to 2 toxicities were fatigue, thrombocytopenia, fever, rash, chills, and anorexia. Reported grade 3 toxicities were hypophosphatemia and grade 3 pruritus. Autoinduction, a phenomenon whereby prolonged administration leads to decreased plasma exposures, has also been observed $[10,11]$.

Our phase I study evaluates RO4929097 in combination with gemcitabine in advanced solid tumors. Gemcitabine is active as monotherapy in many malignancies including pancreatic cancer, non small-cell lung cancer, breast cancer, bladder cancer, ovarian cancer, cervical cancer, head and neck, small cell lung cancer and mesothelioma [12]. A favourable toxicity profile renders gemcitabine amenable to evaluation in combination with targeted agents. Resistance to chemotherapy may be possibly overcome by targeting key pathways to inhibit stem cell propogation; hence the rationale for combining a gamma secretase inhibitor with chemotherapy. Preclinical evidence suggests that gamma secretase inhibition may specifically enhance the antitumor activity of gemcitabine. Cook et al [13] recently evaluated xenograft models treated with either gemcitabine, a gamma secretase inhibitor, or gemcitabine combined with a gamma secretase inhibitor. Although treatment with the gamma secretase inhibitor alone did not reduce tumor volume in this mouse model, combination with gemcitabine prolonged animal survival greater than either gamma secretase inhibition or gemcitabine alone. The data also demonstrated the antivascular effects of gamma secretase inhibition [14], one that was synergistic with the co-administration with gemcitabine, leading to vascular regression and intratumoral hypoxia. This further supports the strategy of using a gamma secretase inhibitor in combination with cytotoxic therapy as in the present phase I study.

We report the results of our phase I study evaluating the oral gamma secretase inhibitor, RO4929097 in combination with gemcitabine in advanced solid tumors. The primary objective was to establish the maximum tolerated dose and recommended phase II dose of RO4929097 in combination with gemcitabine. The secondary objectives were to evaluate safety, tolerability, pharmacokinetics, preliminary anti-tumor activity of RO4929097 in combination with gemcitabine and to explore biomarkers of Notch signaling in archival tumor tissue.

\section{Methods}

Patient selection

Key inclusion criteria were histologically or cytologically proven advanced solid tumors with no further standard treatment options available, age $\geq 18$ years, life expectancy $\geq$ 12 weeks, Eastern Cooperative Oncology Group performance status $\leq 1$, adequate organ and marrow function and the ability to swallow medication. While no limitation on number of prior lines of therapy was made, patients were required to be $\geq 4$ weeks from systemic or radiotherapy and $\geq 6$ weeks from treatment with either nitrosourea or mitomycin $\mathrm{C}$ with the exception of low dose, non-myelosuppressive radiotherapy. Measurable disease was required.

Key exclusion criteria were prior treatment with a Notch inhibitor, known brain metastases (due to poor prognosis and the potential impact on evaluating neurologic adverse events), history of allergic reactions to compounds similar to RO4929097 or gemcitabine, patients taking medications with 
narrow therapeutic indices metabolized by cytochrome (CYP) $\mathrm{P} 450$ or receiving strong CYP3A4 inducers and/or inhibitors for which no medically appropriate alterative was available. Patients with gastrointestinal syndromes, liver disease, known HIV on antiretroviral therapy (due to the potential for pharmacokinetic interactions with RO4929097), uncontrolled electrolyte abnormality, uncontrolled grade $\geq 2$ diarrhea, risk of QT interval prolongation, or coexisting severe medical conditions were excluded. Informed consent was required. This study (PHL-078/CTEP 8575) is conducted by the Princess Margaret Hospital Phase I Consortium with support from the US National Cancer Institute U01 Cooperative Agreement Award (U01-CA132123).

\section{Dose escalation}

A standard $3+3$ dose escalation scheme was used. The recommended phase II dose (RP2D) is the dose level at which $\leq 1$ of 6 patients experience a DLT. Intra-patient dose escalation was not allowed. Patients received a fixed dose of intravenous gemcitabine at $1,000 \mathrm{mg} / \mathrm{m}^{2}$ on days 1,8 and 15 with daily oral dosing of RO4929097 on days $1-3,8-10$, 15-18 and 22-24 (3 on, 4 off continuous schedule) during a 28 day cycle. RO4929097 dosing occurred at approximately the same time of the day, on an empty stomach either $1 \mathrm{~h}$ before a meal or $2 \mathrm{~h}$ following a meal. Pre-planned RO4929097 dose levels were $20 \mathrm{mg}, 30 \mathrm{mg}, 45 \mathrm{mg}$, $90 \mathrm{mg}, 180 \mathrm{mg}$ and $270 \mathrm{mg}$. The rationale for starting dose and scheduling was based on the RP2D of the single agent phase I trial [10]. Treatment continued until unacceptable toxicity, study withdrawal, documented disease progression or until study completion.

\section{Definition of DLT}

DLT was assessed during the first 28-day cycle. DLT was defined as: grade 4 neutropenia lasting $>7$ days, febrile neutropenia, grade 4 thrombocytopenia or thrombocytopenic bleeding, grade $\geq 3$ diarrhea despite management, grade $\geq 3$ electrolyte abnormality unresolved within $72 \mathrm{~h}$, any other grade $\geq 3$ non-hematologic toxicity judged to pose a safety risk, failure to receive $\geq 75 \%$ doses of RO4929097 or $\mathrm{d} 8$ gemcitabine in $\mathrm{C} 1$ due to treatment related toxicity or failure to start $\mathrm{C} 2$ within $\leq 14$ days due to treatment related toxicity.

Study assessments

Standard safety assessments were performed. Toxicity was evaluated according to NCI Common Terminology Criteria for Adverse Events (NCI CTCAE v.4). Radiographic assessment of disease status was performed every 2 cycles and antitumor effect was measured according to RECIST 1.1 criteria [15]. Prolonged stable disease was defined by stable disease for 4 or more months. The data cutoff date for this report was November 6, 2012.

Pharmacokinetic analysis of RO4929097 was performed by collecting serial plasma during cycle 1 on day 1 and day 10 . RO4929097 is highly protein bound and therefore both total and unbound RO4929097 were evaluated [11] Unbound RO4929097 samples were obtained by filtering plasma samples using Amico Centrifree ${ }^{\circledR}$ Micropartition filtration devices (Millipore Corp., Bedford, MA, USA) [16]. Total and unbound RO4929097 concentrations were determined using validated HPLC-tandem mass spectrometry methods. Pharmacokinetic parameters were calculated by nonparametric methods using WinNonlin (Version 5.3, Pharsight Corp., Sunnyvale, CA).

Plasma was collected on day 1 of cycles 1 and 2 for circulating angiogenic factors IL-6, IL-8, VEGF-A, -C, -D, bFGF and SDF-1 alpha. Quantitative analysis was performed using Enzyme-Linked Immunosorbent Assays (ELISA) (R\&D Systems, Minneapolis, MN). The assays were performed in duplicate according to the manufacturer's instructions. Where duplicate values differed by more than $15 \%$, a triplicate was performed. If at least two results were not within $15 \%$ of each other, results were discarded. The association between circulating angiogenic factor levels with grade 3 or more adverse events was evaluated by the Wilcoxon test. The association between circulating angiogenic factor levels with time to progression was evaluated by univariate Cox proportional hazard analysis.

Notch pathway signaling was evaluated using three biomarkers (Jagged1, Notch1- intracellular notch domain [ICD] and Notch3) on archived paraffin-embedded specimens using the Allred scoring system [17-19]. Correlation with outcome was explored. The Wilcoxon rank test was used to compare Notch pathway protein expression from patients with and without either prolonged stable disease or response. Univariate Cox proportional hazard analysis was used to correlate protein expression with time to progression.

\section{Results}

Study population

A total of 18 patients were enrolled at two participating institutions to determine the recommended phase II dose. Of these, 3 patients received $20 \mathrm{mg}$ RO4929097, 7 patients received $30 \mathrm{mg}$ RO4929097, 6 patients received $45 \mathrm{mg}$ RO4929097 and 2 patients received $90 \mathrm{mg}$ RO4929097. First patient, first visit was June 14, 2010. Mean age was 55 years. A median of 3 cycles (range 1-6) per patient was delivered. Breast (5) and pancreatic (3) cancers were well represented. Baseline characteristics for the study population are summarized in Table 1. 
Table 1 Baseline characteristics of enrolled study patients

\begin{tabular}{ll}
\hline & No. of patients \\
\hline Total no. Patients enrolled & 18 \\
Male: Female & $5: 13$ \\
Mean age (Range) & $55(31-76)$ \\
ECOG performance status 0 & 4 \\
ECOG performance status 1 & 14 \\
Tumor type & \\
Breast & 5 \\
Pancreas & 3 \\
Ovary & 2 \\
Nasopharynx & 2 \\
Other & 6 \\
Prior systemic & $4: 3: 3: 4: 2: 1: 1$ \\
therapy regimens $(0: 1: 2: 3: 4: 5: 6)$ & \\
\hline
\end{tabular}

Dose escalation

Three patients were enrolled in dose level 1 (20 mg RO4929097). No dose limiting toxicities were observed. Of the three patients enrolled in the next dose level $(30 \mathrm{mg})$, one dose limiting toxicity of grade 3 transaminitis was observed. The dose level was expanded to 6 patients at $30 \mathrm{mg}$ with no further dose limiting toxicities. One patient at the $30 \mathrm{mg}$ dose level discontinued study due to progressive disease causing perforated viscous and death; this patient was non-evaluable for toxicity and was replaced. The first 3 patients at dose level 3 , (45 mg) had no dose limiting toxicities, thus the dose was escalated to $90 \mathrm{mg}$ where there were 2 dose limiting toxicities. One patient experienced grade 3 transaminitis. The second patient failed to receive $75 \%$ of planned RO4929097 doses secondary to prolonged neutropenia. Dosing was then deescalated to $45 \mathrm{mg}$ RO4929097 and 3 additional patients were treated. In this cohort, 2 dose limiting toxicities were observed. One patient experienced a grade 3 transaminitis. The second patient experienced a grade 3 maculopapular rash. Observed dose limiting toxicities are summarized in Table 2. The recommended phase II dose is $30 \mathrm{mg}$ orally in a 3 day on, 4 day off, continuous schedule in combination with

Table 2 Observed dose limiting toxicities of RO4929097 in combination with gemcitabine

\begin{tabular}{|c|c|c|c|c|c|}
\hline Cohort & $\begin{array}{l}\text { Dose } \\
\text { level }\end{array}$ & $\begin{array}{l}\text { No. Patients } \\
\text { in DL }\end{array}$ & $\begin{array}{l}\text { No. } \\
\text { DLTs }\end{array}$ & Event & Grade \\
\hline 1 & $1(20 \mathrm{mg})$ & 3 & 0 & & \\
\hline 2 & $2(30 \mathrm{mg})$ & 7 & 1 & Transaminitis & 3 \\
\hline \multirow[t]{2}{*}{3} & $3(45 \mathrm{mg})$ & 6 & 2 & Transaminitis & 3 \\
\hline & & & & Maculopapular rash & 3 \\
\hline \multirow[t]{2}{*}{4} & $4(90 \mathrm{mg})$ & 2 & 2 & Transaminitis & 3 \\
\hline & & & & $<75 \%$ RO doses & 3 \\
\hline
\end{tabular}

intravenous gemcitabine dosed at $1,000 \mathrm{mg} / \mathrm{m}^{2}$ on days 1 , 8 and 15 every 4 weeks.

Tolerability

Common toxicities at least possibly related to RO4929097 occurring in $10 \%$ or more of patients included nausea, fatigue, anorexia, vomiting, diarrhea, hypophosphatemia, hypomagnesemia, rash and transaminitis. The frequency and grade of common toxicities are summarized in Table 3. Hypophosphatemia, transaminitis and rash were the most common (frequency $10 \%$ or more) grade 3 adverse events. The remaining toxicities were mild. All toxicities were reversible with supportive measures, and interruption of study drug.

Pharmacokinetics

Pharmacokinetic analysis for total and free RO4929097 is shown in Table 4. Pharmacokinetic analysis demonstrated that repeated oral dosing of RO4929097 resulted in increased exposures at increased doses. To evaluate the presence of autoinduction, day 10:day 1 AUC 0-24 levels were compared for both total and free RO4929097. In the absence of autoinduction, day 10 levels are expected to equal or exceed day 1 levels due to accumulation. Evaluation of Day 10: Day 1 total and free RO4929097 levels confirmed the presence of autoinduction at dose levels 45 and $90 \mathrm{mg}$ (Fig. 1a-b).

Circulating angiogenic factors

Quantitative ELISA analysis of circulating angiogenic factors IL-6, IL-8, VEGF-A, -C, -D, bFGF was performed on serum samples collected on day 1 of cycles 1 and 2 . Circulating angiogenic factor levels were not associated with the occurrence of grade 3 or higher adverse events or time to progression (data not shown).

Table 3 Common observed toxicities occurring in $10 \%$ or more of patients at least possibly related to RO4929097

\begin{tabular}{lll}
\hline Event & $\begin{array}{l}\text { Frequency (n) } \\
\text { Any grade }\end{array}$ & $\begin{array}{l}\text { Frequency (n) } \\
\text { Grade } \geq 3\end{array}$ \\
\hline Nausea & $61 \%(11)$ & 0 \\
Fatigue & $56 \%(10)$ & 0 \\
Vomiting & $39 \%(7)$ & 0 \\
Hypophosphatemia & $33 \%(6)$ & $6 \%(1)$ \\
Anorexia & $22 \%(4)$ & 0 \\
Transaminitis & $22 \%(4)$ & $6 \%(1)$ \\
Maculopapular rash & $17 \%(3)$ & $6 \%(1)$ \\
Hypomagnesemia & $11 \%(2)$ & 0 \\
Acneiform rash & $11 \%(2)$ & 0 \\
Diarrhea & $11 \%(2)$ & 0 \\
\hline
\end{tabular}


Table 4 Pharmacokinetic analysis of total and free RO4929097. Sample for 1 patient in DL 2 was not available for analysis. Cmax (ng/ml); AUC $0-24$ $\left(\mathrm{ng} \times \mathrm{hr} / \mathrm{ml} \times 10^{4}\right)$. Standard deviations in brackets

\begin{tabular}{|c|c|c|c|c|c|c|c|c|c|}
\hline DL & $\mathrm{n}$ & $\begin{array}{l}\text { Total D1 } \\
\text { Cmax }\end{array}$ & $\begin{array}{l}\text { Total D10 } \\
\text { Cmax }\end{array}$ & $\begin{array}{l}\text { Free D1 } \\
\text { Cmax }\end{array}$ & $\begin{array}{l}\text { Free D10 } \\
\text { Cmax }\end{array}$ & $\begin{array}{l}\text { Total D1 } \\
\text { AUC }_{0-24}\end{array}$ & $\begin{array}{l}\text { Total D10 } \\
\text { AUC } 0-24\end{array}$ & $\begin{array}{l}\text { Free D1 } \\
\text { AUC } 0-24\end{array}$ & $\begin{array}{l}\text { Free D10 } \\
\text { AUC }_{0-24}\end{array}$ \\
\hline 1 & 3 & $552(100)$ & $615(382)$ & $46.7(7.6)$ & $42.1(23.3)$ & $6.8(1.0)$ & $9.9(7.2)$ & $540(60.0)$ & $586(362)$ \\
\hline 2 & 6 & $833(352)$ & $1221(315)$ & $54.7(20.3)$ & $68.1(15.0)$ & $12.6(4.8)$ & $19.2(5.9)$ & $644(295)$ & $990(202)$ \\
\hline 3 & 6 & $986(153)$ & $1315(332)$ & $71.6(30.6)$ & $72.3(13.1)$ & $12.8(1.2)$ & $16.5(8.2)$ & $924(369)$ & $1687(157)$ \\
\hline 4 & 2 & $1635(742)$ & $1205(290)$ & $107.5(36.0)$ & $80.7(28.7)$ & $6.8(1.0)$ & $9.9(7.2)$ & $1697(875)$ & $962(670)$ \\
\hline
\end{tabular}

Notch signaling biomarkers

Jagged1, N1-ICD and Notch3, were evaluated by immunohistochemistry in all available archival paraffin embedded patient samples. Immunohistochemical staining scores for median Notch3 scores were significantly higher in individuals who received fewer than 4 cycles (score 7) compared to those

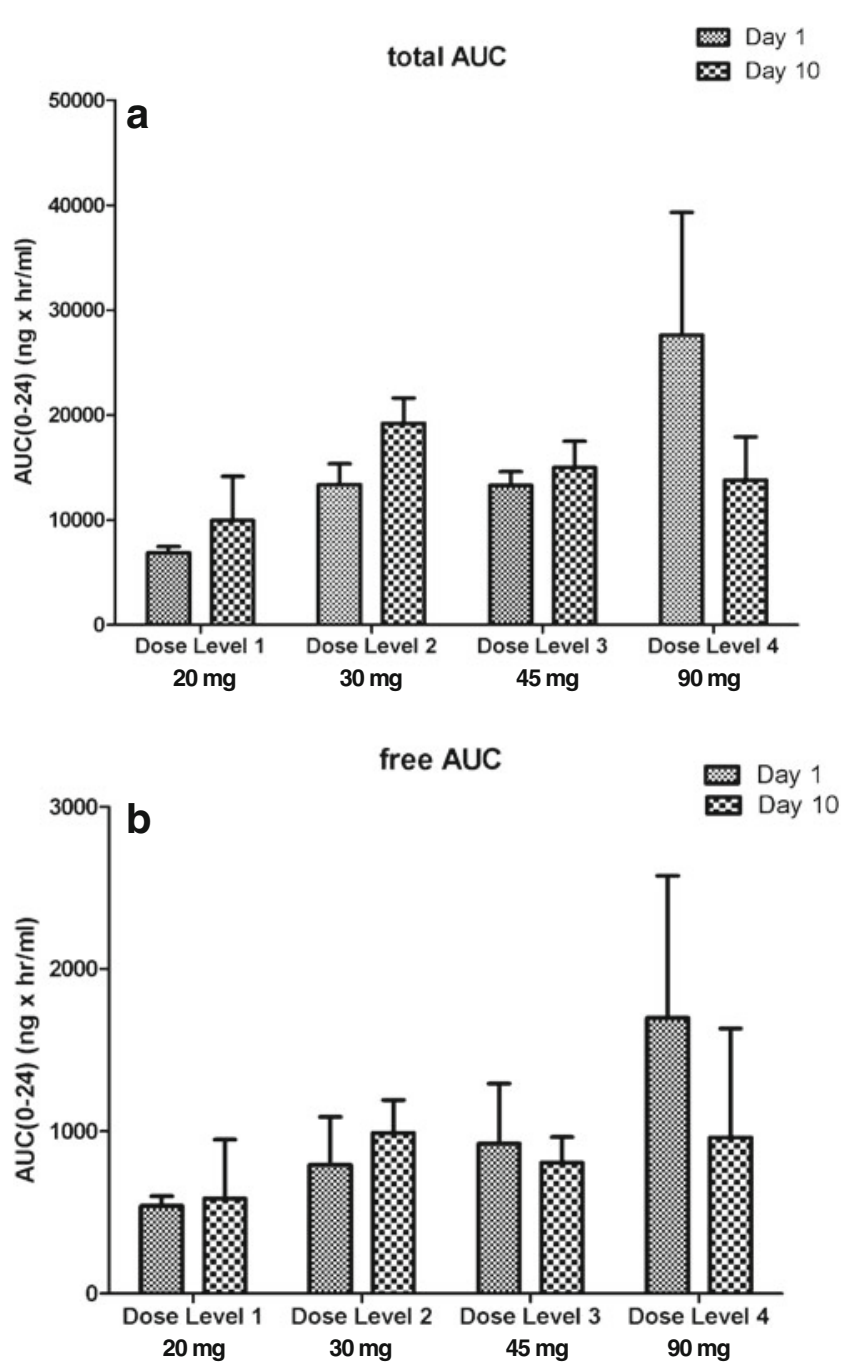

Fig. 1 a day 10: day 1 area under the curve 0-24 for total RO4929097. b day 10: day 1 area under the curve 0-24 for free RO4929097 individuals who received 4 cycles of treatment of more (score 5) $(p=0.029)$ (Fig. 2). For Jagged1 and N1-ICD, there were no significant differences in staining scores identified when samples from patients receiving fewer than 4 cycles were compared to those who received 4 or more cycles of treatment. Univariate Cox proportional hazard ratios for time to progression and Jagged1, N1-ICD and Notch3 were not statistically significant.

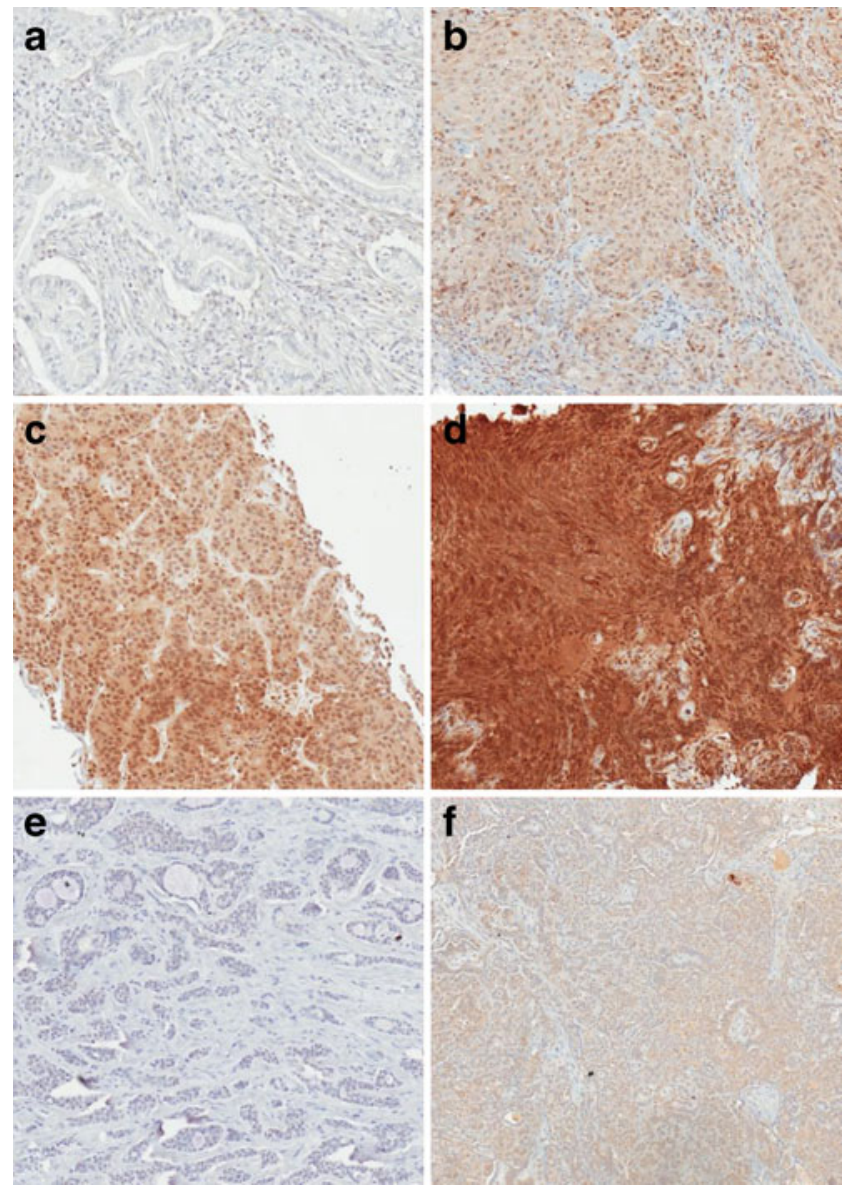

Fig. 2 Immunohistochemical staining for Notch Proteins a. Absence of Notch 3 staining in oropharyngeal adenocarcinoma $\mathbf{b}$. Weak diffuse Notch 3 staining in squamous cell carcinoma c. Intermediate diffuse staining for Notch 3 in metastatic breast ductal carcinoma d. Strong diffuse staining for Notch 3 nasopharyngeal squamous cell carcinoma e. Absence of staining for Notch 1- intracellular notch domain in tracheal adenoid cystic carcinoma f. Weak staining for Jagged 1 in low grade serous carcinoma of ovary 
Antitumor activity

Preliminary evidence of antitumor activity was observed for RO4929097 in combination with gemcitabine. A partial response was observed in one patient with nasopharyngeal carcinoma who was enrolled at the $45 \mathrm{mg}$ dose level. Prolonged stable disease was observed in one patient with pancreatic cancer, one patient with tracheal cancer and one patient with cervical cancer (Fig. 3).

\section{Discussion}

We report the results of our phase I study of RO4929097, an oral gamma secretase inhibitor, in combination with gemcitabine in adult patients with advanced solid tumors. The recommended phase II dose is $30 \mathrm{mg}$ orally in a 3 day on, 4 day off, continuous schedule in combination with intravenous gemcitabine dosed at $1,000 \mathrm{mg} / \mathrm{m}^{2}$ on days 1 , 8 and 15 every 4 weeks.

The combination of RO4929097 and gemcitabine was tolerable, albeit with increasing toxicity at increasing dose levels. Common observed toxicities included nausea, fatigue, diarrhea, hypomagnesemia, hypophosphatemia and transaminitis. No new toxicities were identified. These effects are similar to those identified with RO4929097 evaluated as monotherapy in a phase I trial [10], in combination with capecitabine [20], and are also similar to those observed with other gamma secretase inhibitors [21]. The intestinal goblet cell toxicity observed in preclinical models has not proven to be a dose limiting toxicity in the clinical evaluation of RO4929097. This may be due to the specific RO4929097 scheduling using intermittent dosing on a 3 on, 4 off schedule or the lack of target engagement.

Efficacy results demonstrated response in one patient with nasopharyngeal carcinoma and prolonged stable disease in 3

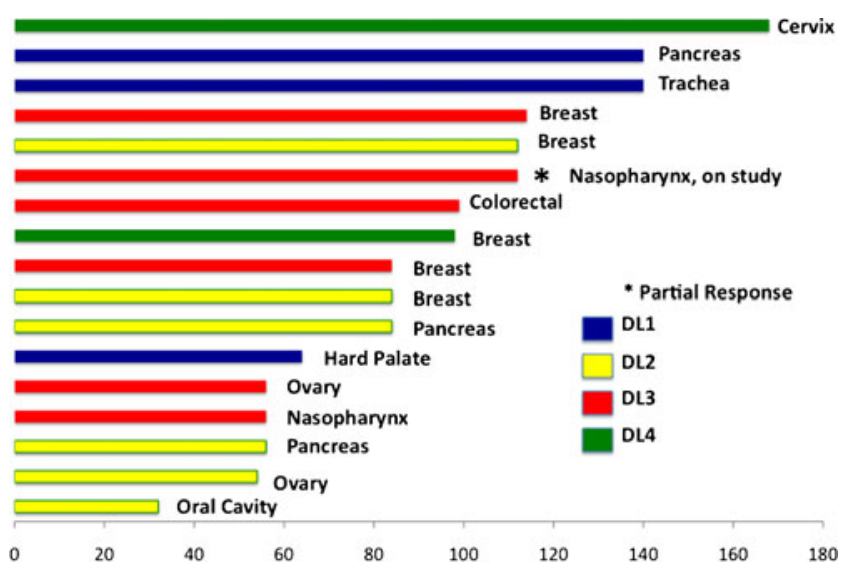

Fig. 3 Antitumor activity of R04929097 in combination with gemcitabine. Days on treatment are shown along the $\mathrm{x}$ axis patients, one with each of pancreatic, tracheal and cervical cancer. Patients with pancreatic cancer were specifically recruited due to the evidence linking a strong role for Notch signaling in pancreatic tumors [13]. The expected antitumour activity due to Notch inhibition alone may be cytostatic. This is supported by in vitro data demonstrating that RO4929097 treatment produced a more flattened phenotype, rather than overt cell death, as well as by data showing that combination treatment with RO4929097 and gemcitabine in a mouse xenograft model of pancreatic cancer led to prolonged survival despite the lack of decrease in tumor volume. In our study, patients with prolonged stable disease may have experienced some benefit from RO4929097 therapy though confirmation of this would require a randomized controlled study. Furthermore, the additive impact of the investigational agent RO4929097, with gemcitabine, a conventional cytotoxic chemotherapy with efficacy in pancreatic and nasopharyngeal carcinomas, cannot be delineated in this single arm phase I study.

While the exposures of RO4929097 achieved exceeded the active levels identified in preclinical models, autoinduction was observed starting at levels as low $45 \mathrm{mg}$. The intermittent scheduling of 3 on, 4 off dosing was considered to minimize the autoinduction observed in previous studies. However, in our study, pharmacokinetic analysis of day 10: day 1 areas under the curve for RO4929097 decreased below 1 with increasing dose levels. These results are consistent with the effect of autoinduction on RO4929097 metabolism with the evaluated dose levels. As dose was increased over $45 \mathrm{mg}$, autoinduction became more prominent. This pharmacokinetic property of RO4929097 has been observed in another study, with a minimum of 7 days of discontinuation required to return to baseline kinetics [10]. Autoinduction presents a challenge for both dose titration and the potential to combine RO4929097 with agents metabolized by the liver.

We acknowledge some of the limitations of our pharmacokinetic analysis; given that the half-life of R04929097 is approximately $20 \mathrm{~h}$, we would require 4 to 5 half-lives of pharmacokinetic sampling in order to accurately calculate AUC 0-inf. We based our pharmacokinetic sampling schedule on previous reports [9]; thus day 10:day1 ratios were used with the assumption that day 10 exposures are expected to be higher than day 1 exposures serves as a surrogate marker for evaluating the presence of autoinduction. It is on the basis of this definition that we identified the presence of autoinduction at 45-90 mg of RO4929097 in this study.

Exploratory correlatives were analyzed to further evaluate the pharmacodynamics of RO4929097. Correlative studies demonstrated that higher levels of Notch3 protein expression in archival tissue were associated with resistance to treatment of RO4929097 in combination with gemcitabine in this study. While this relationship is interesting and consistent with previous reports [22], it is an exploratory finding in this single arm study designed primarily for dose finding. The 
significance of Notch amplification as a mechanism of resistance may be further evaluated in a large randomized study.

The Notch axis is implicated in angiogenesis in conjunction with vascular endothelial growth factor signaling [14]. In our study with a small number of responders, levels of circulating angiogenic factors did not show correlation between time to progression or statistically significant antiangiogenic effects of RO4929097. This observation may be due to the effect of autoinduction on decreasing steady state levels or alternatively the absence of specific antiangiogenic drug effects. Treatment with RO4929097 has been shown to reduce the expression of circulating angiogenesis factors in vitro $[9,23]$. Further studies are needed to further evaluate the clinical antiangiogenic effects of Notch axis signaling.

In summary, the data from this phase I study demonstrates that the oral gamma secretase inhibitor, RO4929097, can be safely combined with gemcitabine in adult patients with advanced solid tumors. This is the first study to report the combination of a notch inhibitor with intravenous chemotherapy. The recommended phase II dose is $30 \mathrm{mg}$ using a 3 day on, 4 day off dosing schedule. While the exposures of RO4929097 achieved exceeded the active levels identified in preclinical models, autoinduction was observed starting at levels as low $45 \mathrm{mg}$, limiting further titration. Antitumor activity was seen in several tumor types including pancreas and nasopharyngeal carcinoma, although activity due to gemcitabine alone could not be excluded. Notch protein expression was lower in patients who achieved prolonged stable disease or partial response, however evidence of target engagement was not identified by analysis of circulating angiogenic factor levels. Due to the discontinuation of RO4929097 drug development programs, a planned expansion at the RPTD in a pancreatic cancer cohort did not proceed. Further studies with other gamma secretase inhibitors in combination with chemotherapy will be needed to expand the role of this strategy in advanced solid tumours.

Conflicts of Interest The authors declare that they have no conflict of interest.

Open Access This article is distributed under the terms of the Creative Commons Attribution License which permits any use, distribution, and reproduction in any medium, provided the original author(s) and the source are credited.

\section{References}

1. Miele L, Miao H, Nickoloff BJ (2006) NOTCH signaling as a novel cancer therapeutic target. Curr Cancer Drug Targets 6:313-323

2. Bolos V, Grego-Bessa J, de la Pompa JL (2007) Notch signaling in development and cancer. Endocr Rev 28:339-363

3. Jarriault S, Le Bail O, Hirsinger E, Pourquie O, Logeat F, Strong CF et al (1998) Delta-1 activation of Notch-1 signaling results in HES-1 transactivation. Mol Cell Biol 18(12):7423-7431
4. Klinakis A, Szabolcs M, Politi K, Golde T, Osborne B, Miele L (2006) Myc is a Notch1 transcriptional target and a requisite for Notch1-induced mammary tumorigenesis in mice. Proc Natl Acad Sci U S A 103:9262-9267

5. Rizzo P, Osipo C, Foreman K et al (2008) Rationale targeting of Notch signaling in cancer. Oncogene 27:5124-5131

6. Dufraine J, Funahashi Y, Kitajewski J (2008) Notch signaling regulates tumor angiogenesis by diverse mechanisms. Oncogene 27:5132-5137

7. Phng LK, Gerhardt H (2009) Angiogenesis: a team effort coordinated by notch. Dev Cell 16:196-208

8. Plentz R, Park JS, Rhim AD, Abravanel D, Hezel AF, Sharma SV et al (2009) Inhibition of gamma-secretase activity inhibits tumor progression in a mouse model of pancreatic ductal adenocarcinoma. Gastroenterology 136:1741-1749 e6

9. Luistro L, He W, Smith M, Packman K, Vilenchik M, Carvajal D et al (2009) Preclinical profile of a potent gamma-secretase inhibitor targeting notch signaling with in vivo efficacy and pharmacodynamic properties. Cancer Res 69:7672-7680

10. Tolcher A, Messersmith W, Mikulski SM, Papadopoulos KP, Kwak EL, Gibbon DG et al (2012) Phase I study of RO4929097, a gamma secretase inhibitor of Notch signaling, in patients with refractory metastatic or locally advanced solid tumors. J Clin Oncol 30:2348-2353

11. Wu J, Lorusso PM, Matherly LH, Li J (2012) Implications of plasma protein binding for pharmacokinetcs and pharmacodynamics of the g-secretase inhibitor RO4929097. Clin Cancer Res 18(7):2066-2079

12. Van Moorsel C, Peters GJ, Pinedo HM (1997) Gemcitabine: future prospects of single-agent and combination studies. Oncologist 2:127-134

13. Cook N, Frese KK, Bapiro TE, Jacobetz MA, Gopinathan A, Miller JL (2012) Gamma secretase inhibition promotes hypoxic necrosis in mouse pancreatic ductal adenocarcinoma. J Exp Med 209(3):437-444

14. Carmeliet P, DeSmet F, Loges S, Mazzone M (2009) Branching morphogenesis and antiangiogenesis candidates: tip cells lead the way. Nat Rev Clin Oncol 6:315-e326

15. Eisenhauer EA, Therasse P, Bogaerts J, Schwartz LH, Sargent D, Ford R et al (2009) New response evaluation criteria in solid tumors: revised RECIST guideline (version 1.1). Eur J Cancer 45:228-247

16. Zhang W, Seymour L, Chen EX (2008) Determination of intact oxaliplatin in human plasma using high performance liquid chromatography-tandem mass spectrometry. J Chromatography B 876:277-282

17. Allred DC, Harvey JM, Berardo M, Clark GM (1998) Prognostic and predictive factors in breast cancer by immunohistochemical analysis. Mod Pathol 11(2):155-168

18. Dickson BC, Mulligan AM, Zhang H, Lockwood G, O’Malley FP, Egan SE et al (2007) High-lvel JAG1 mRNA and protein predict poor outcome in breast cancer. Mod Pathol 20:685-693

19. Reedijk M, Pinnaduwage D, Dickson BC, Mulligan AM, Zhang H, Bull SB et al (2008) JAG1 expression is associated with a basal phenotype and recurrence in lymph node-negative breast cancer. Breast Cancer Res Treat 111:439-448

20. Strosberg JR, Yeatman T, Weber J, Coppola D, Schell MJ, Han G et al (2012) A phase II study of RO4929097 in metastatic colorectal cancer. Eur J Cancer 48(7):997-1003

21. Krop I, Demuth T, Guthrie T, Wen PY, Mason WP, Chinnaiyan P et al (2011) Phase I pharmacologic and pharmacodynamic study of the gamma secretase (Notch) inhibitor MK-0752 in adult patients with advanced solid tumors. J Clin Oncol 30(19):2307-2313

22. Dang TP, Gazdar AF, Virmani AK, Sepetavec T, Hande KR, Minna JD et al (2000) Chromosome 19 translocation, overexpression of Notch3, and human lung cancer. J Natl Cancer Inst 92:1355-e1357

23. He W, Luistro L, Carvajal D, Smith M, Nevins T, Yin X et al (2011) High tumor levels of IL6 and IL8 abrogate preclinical efficacy of the g-secretase inhibitor, RO4929097. Mol Oncol 5(3):292-301 